$4.1 \%$ mortality found in a similar analysis of patients in 2001 in the USA. ${ }^{2}$

Statistically significant risk factors for patients undergoing lung resection for cancer included male gender, increasing age, operations on the right lung and the need for more extensive operations such as pneumonectomy or bilobectomy. In addition, patients with a higher Charlson co-morbidity index had a higher risk of postoperative mortality than patients with a lower index. These findings were used to generate a prediction model which can be used to define the risk of 30-day postoperative mortality for any specific patient. Knowing these risk factors and having the risk formula will be useful for thoracic surgeons and provide the opportunity for an evidence-based selection of patients for surgery. However, an important caveat which the authors make is that "... surgical treatment should not be withheld because of co-morbid conditions ... alone". In other words, there is still need for art as well as science.

Some potential risk factors were not conclusively defined. First, the relationship between surgeon specialty and outcomes could not be identified any more specifically than to say that some were general surgeons and some were cardiothoracic surgeons. This relationship, of course, would be interesting to know as previously reported studies have found better outcomes for dedicated thoracic surgeons. ${ }^{3}{ }^{4}$ Two other risk and outcome considerations which have been the target of inconclusive international debate could not be definitively resolved by this study ${ }^{5}$-namely, the type of hospital (general vs university) and the question of hospital volume. The $\mathrm{p}$ values for both these analyses approached but did not reach statistical significance. The question therefore remains of the possibility of a $\beta$-type error and that, with a larger dataset, both of these differences might in fact reach statistical significance. The issue is also clouded by the fact that most of the Norwegian highvolume hospitals were also university hospitals, confounding the issue even further. The questions of the roles of these possible determinants of outcome, surgeon specialisation, hospital type and hospital volume remain unsettled.

This influential study emphatically highlights the value and importance of tracking patient outcomes in a prospective database. This report and its observations would not have been possible without the government-required Cancer Registry of Norway. In the USA the two equivalents are the Society of Thoracic Surgeons database and the American College of Surgeons' national cancer database. Participation in these databases allows individual surgeons, surgical groups and hospitals to compare their outcomes with national outcomes which, in turn, allow them to identify areas where performance and quality can and should be improved. Further, these large databases allow determination of risk adjusted mortality and morbidity rates, which are the only legitimate and reasonable way to compare, stratify and determine appropriate outcomes.

One factor not considered by this report is the distinction between the length of life and its quality. For any comprehensive consideration of the risk-benefit of lung resection for patients with lung cancer, follow-up beyond 30 days and, in fact, for a lifetime is therefore essential. If the patient survives but experiences severe post-thoracotomy pain, becomes a respiratory cripple, or is unable to resume the desired lifestyle, the "benefit" of a curative operation is compromised. The complete picture must be kept in mind. Quality of life, as well as its length, is an essential consideration in developing and recommending therapeutic strategies. ${ }^{6}$

Thorax 2007;62:929-930.

doi: 10.1136/thx.2007.082982

Correspondence to: Dr Alex G Little, Wright State University, Boonshoft School of Medicine,

Department of Surgery, 1 Wyoming Street, Suite 7801 WCHE, Dayton, Ohio 45409, USA; alex. little@wright.edu

Competing interests: None.

\section{REFERENCES}

1 Strand T-E, Rostad H, Dambuis RAM, et al. Risk factors for 30-day mortality after resection of lung cancer and prediction of their magnitude. Thorax 2007;62:991-7.

2 Little AG, Rusch WW, Bonner JA, et al. Patterns of surgical care of lung cancer patients. Ann Thorac Surg 2005;80:2051-6.

3 Silvestri GA, Handy J, Lackland D, et al. Specialists achieve better outcomes than generalists for lung cancer surgery. Chest 1998;114:675-80.

4 Goodney PP, Lucas FL, Stuckel TA, et al. Surgeon specialty and operative mortality with lung resection. Ann Surg 2005;241:179-84.

5 Bach PB, Cramer LD, Schrag D, et al. The influence of hospital volume on survival after resection for lung cancer. N Engl J Med 2001;345:181-8.

6 Paull DE, Thomas ML, Meade GE, et al. Determinants of quality of life in patients following pulmonary resection for lung cancer. Am J Surg 2006;192:565-71.

\title{
Nicotine replacement therapy for smoking cessation in the "real world"
}

\section{Saul Shiffman}

\section{NRT works in "real would" settings, but optimising its use would improve its impact on public health}

$\mathrm{T}$ he paper by West and Zhou published in this issue of Thorax (see page 998)

is an important contribution to the literature because it addresses and refutes the questions raised, on the basis of retrospective case-control studies, about the effectiveness of nicotine replacement therapy (NRT) in "real world" settings. ${ }^{12}$
Contrary to those retrospective analyses, West and Zhou's prospective population study found that NRT helps smokers to quit, roughly doubling the odds of successful quitting. The odds ratio of 2.2 reported by West and Zhou is quite consistent with the odds ratios reported in randomised clinical trials. ${ }^{3}$
In this sense, West and Zhou's finding that NRT is effective should come as no surprise. The efficacy of NRT has been demonstrated in over 100 randomised controlled trials encompassing over 35000 smokers. $^{3}$ Moreover, the effectiveness of NRT has also been demonstrated in multiple trials that tested NRT under overthe-counter conditions. ${ }^{4}$ These studies not only showed that over-the-counter NRT was more effective than placebo, but also that it was just as effective as using NRT under the care of a doctor. Although critics have argued that "effectiveness" (in contrast to "efficacy") has not been shown, in fact these trials were effectiveness trials: there was little or no screening or exclusion of participants, no extra support, instruction or therapy offered, and minimal data collection. ${ }^{5}$ The benefit of NRT has therefore been proven in both efficacy and effectiveness studies. West and Zhou's data extend this to a prospective study of 
population samples not enrolled in a treatment trial, and thus help to address the concern that subjects enrolled in treatment trials may not be representative of the population.

Why are the analyses from retrospective surveys, which appear to show little or no efficacy either for NRT or for any behavioural treatment, ${ }^{267}$ so discrepant with the findings of West and Zhou and those of dozens of other studies? One important reason, alluded to by West and Zhou, is that brief failed quit attempts are easily forgotten unless something makes them memorable. ${ }^{8}$ Spending money on an NRT product (or being stuck with acupuncture needles, for that matter) is unusual and therefore memorable, with the likely result that failures on NRT are recalled and reported while failures in unaided quitting are forgotten or perhaps dismissed as not being "real" quit attempts. This can create substantial bias in retrospective reporting of quit success, making treatments of all kinds appear ineffective and making retrospective surveys unsuitable for evaluating effectiveness.

Another important bias in uncontrolled population studies of cessation methods is that smokers self-select which method they use for quitting. West and Zhou's data confirm the findings from other studies that more dependent smokerswho have a lower probability of success in the first place-gravitate towards treatment. ${ }^{9}$ This artificially depresses success rates in the treatment group compared with the self-selected non-treaters. West and Zhou try to control for self-selection by including in their models a single measure of nicotine dependence. It is unlikely, however, that this completely controls for this "indication bias"; 10 the realisation by smokers that they need help to quit smoking is based on a host of additional factors that were not controlled for in these analyses such as low self-efficacy and lack of social support. ${ }^{9}$ This suggests that the treatment effect reported by West and Zhou probably understates the actual benefit of treatment and again highlights the value of randomised trials for obtaining unbiased estimates of treatment effects.
While West and Zhou's data show that NRT is effective even when used without support or behavioural treatment in an over-the-counter setting, this is not the optimal use of NRT. There is ample evidence that behavioural intervention can further additively improve success rates for smokers using NRT, ${ }^{31}$ so the optimal approach for any smoker is to use NRT and behavioural treatment. Unfortunately, very few smokers use behavioural treatments, ${ }^{6}{ }^{12}$ even though behavioural support is increasingly available free of charge through convenient channels such as telephone quitlines ${ }^{13}$ and internet programs. ${ }^{11}$ All smokers should be encouraged to get behavioural treatment.

The way NRT itself is used is also often not optimal. Smokers who use NRT too often fail to follow the prescribed regimen: they use too little medication ${ }^{14}$ and use it for too short a time, ${ }^{15}$ both of which reduce the effectiveness of the medication. ${ }^{16}{ }^{17}$ One reason smokers under-dose is that too many of them mistakenly believe that nicotine medications are unsafe-in fact, many believe NRT is as dangerous as cigarettes. ${ }^{18}$ We need to do more to educate smokers and to encourage them to use the dose and duration recommended on the label. But the biggest source of NRT "under-dosing" is those smokers who don't use NRT at all. West and Zhou report that about two-thirds of their sample did not use NRT or other treatment in their attempts to quit, which is consistent with recent US data on NRT use. ${ }^{6}$ Those twothirds fail to take advantage of an accessible, safe and effective treatment that, even when used as an over-the-counter product, can double their odds of quitting smoking and possibly save their lives.

Thorax 2007;62:930-931.

doi: 10.1136/thx.2007.081919

Correspondence to: Dr Saul Shiffman, University of Pittsburgh, 130 N. Bellefield Ave (Suite 510), Pittsburgh, Pennsylvania 15213, USA; shiffman@ pitt.edu

Dr Shiffman serves as a consultant to GlaxoSmithKline Consumer Healthcare (GSKCH) on an exclusive basis regarding matters relating to smoking cessation, and also has a financial interest in a venture to develop a new nicotine replacement medication.

\section{REFERENCES}

1 West R, Zhou X. Is nicotine replacement therapy for smoking cessation effective in the "real world"? Findings from a prospective multinational cohort study. Thorax 2007;62:998-1002.

2 Pierce JP, Gilpin EA. Impact of over-the-counter sales on effectiveness of pharmaceutical aids for smoking cessation. JAMA 2002;288:1260-4.

3 Silagy C, Lancaster T, Stead L, et al. Nicotine replacement therapy for smoking cessation. In: Cochrane Library, Issue 1. Chichester: John Wiley \& Sons Ltd, 2004.

4 Hughes JR, Shiffman S, Callas P, et al. A metaanalysis of the efficacy of over-the-counter nicotine replacement. Tob Control 2003;1:21-7.

5 Baver MS, Williford WO, Dawson EE, et al. Principles of effectiveness trials and their implementation in VA Cooperative Study \#430: reducing the efficacy-effectiveness gap in bipolar disorder. J Affect Disord 2001;67:61-78.

6 Shiffman S, Brockwell S, Pillitteri JL, et al. Quit attempts and use of smoking cessation treatments in the USA: an analysis of the 2003 current population survey. Poster presented at the Annual Meeting of the Society for Research on Nicotine and Tobacco; February 2007, Austin, Texas, USA.

7 White P, Watt J. Tobacco control in London: facts and issues. London: SmokeFree London, 2002.

8 Gilpin E, Pierce JP. Measuring smoking cessation: problems with recall in the 1990 California Tobacco Survey. Cancer Epidemiol Biomarkers Prev 1994;3:613-7.

9 Shiffman S, Di Marino ME, Sweeney CT. Characteristics of selectors of nicotine replacement therapy. Tob Control 2005; 14:346-55.

10 Miettinen OS. The need for randomization in the study of intended effects. Stat Med 1983;2:267-71.

11 Strecher VJ, Shiffman S, West R. Randomized controlled trial of a web-based computer-tailored smoking cessation program as a supplement to nicotine patch therapy. Addiction 2005; 100:682-8.

12 Cokkinides VE, Ward E, Jemal A, et al. Under-use of smoking-cessation treatments: Results from the National Health Interview Survey, 2000. Am J Prev Med 2005;28:119-22.

13 Zhu S, Anderson CM, Tedeschi GJ, et al. Evidence of real-world effectiveness of a telephone quitline for smokers. N Engl J Med 2002;347:1087-93.

14 Shiffman S, Rolf CN, Hellebusch SJ, et al. Realworld efficacy of prescription and over-the-counter nicotine replacement therapy. Addiction 2002;97:505-16.

15 Shiffman S, Hughes JR, Pillitteri JL, et al. Persistent use of nicotine replacement therapy: an analysis of actual purchase patterns in a population-based sample. Tob Control 2003;12:310-6.

16 Shiffman S. Use of more nicotine lozenges leads to better success in quitting smoking. Addiction 2007;102:809-14.

17 Shiffman S, Brockwell S, Gitchell J, et al. Wearing nicotine patches continuously for three weeks during a cessation attempt improves short-term efficacy. Poster presented at the Annual Meeting of the Society for Research on Nicotine and Tobacco; February 2007, Austin, Texas, USA.

18 Bansal MA, Cummings KM, Hyland A, et al. Stopsmoking medications: who uses them, who misuses them, and who is misinformed about them? Nicotine Tob Res 2004;6(Suppl 3):S303-10. 